\title{
A synchrotron self-Compton scenario for the very high energy $\gamma$-ray emission of the radiogalaxy M 87
} Unifying the TeV emission of blazars and other AGNs?

\author{
J.-P. Lenain ${ }^{1}$, C. Boisson ${ }^{1}$, H. Sol ${ }^{1}$, and K. Katarzyński ${ }^{2}$ \\ 1 LUTH, Observatoire de Paris, CNRS, Université Paris Diderot; 5 Place Jules Janssen, 92190 Meudon, France \\ e-mail: jean-philippe.lenain@obspm.fr \\ 2 Torun Centre for Astronomy, Nicolaus Copernicus University, ul. Gagarina 11, 87100 Toruń, Poland
}

Received 1 June 2007 / Accepted 12 October 2007

\begin{abstract}
Context. M 87 is the first extragalactic source detected in the TeV range that is not a blazar. With the increasing performances of ground-based Čerenkov telescopes, we can now probe the variability in the $\gamma$-ray flux on small timescales, thus putting strong constraints on the size of the emitting zone. The large scale jet of M 87 is misaligned with respect to the line of sight. A modification of standard emission models of TeV blazars appears necessary to account for the $\gamma$-ray observations despite this misalignment.

Aims. We explain TeV $\gamma$-ray spectra and the fast variability of M 87 by invoking an emission zone close to the central supermassive black hole, which is filled with several plasma blobs moving in the large opening angle of the jet formation zone.

Methods. We develop a new multi-blob synchrotron self-Compton (SSC) model with emitting blobs set on a cap beyond the Alfvén surface in the jet, at a distance of $\sim 100 r_{g}$ from the central engine to interpret the high energies inferred by new TeV observations. We present an SSC model that is explicitly adapted to dealing with large viewing angles and moderate values of the Lorentz factor inferred from (general relativistic) magnetohydrodynamic models of jet formation.

Results. This scenario can account for the recent $\gamma$-ray observations of M 87 made by the High Energy Stereoscopic System (HESS) telescope array. We find individual blob radii of about $10^{14} \mathrm{~cm}$, that is compatible with the variability on timescales of days recently reported by the HESS collaboration and is close to the black hole gravitational radius. Predictions of the very high-energy emission for three other sources with extended optical or X-ray jet that could be misaligned blazars still with moderate beaming are presented for one Seyfert 2 radiogalaxy, namely Cen A, one peculiar BL Lac, PKS 0521-36, and one quasar, 3C 273.
\end{abstract}

Key words. galaxies: active - galaxies: individual: M 87 - gamma rays: theory - radiation mechanisms: non-thermal

\section{Introduction}

M 87 is a well-known, nearby giant elliptical galaxy $(z=$ 0.00436 , Smith et al. 2000) close to the center of the Virgo cluster, which shows a multi-spectral jet, the signature of an active galactic nucleus (AGN). Its jet is one of the best known, on all scales, thanks to its nearby location and its strong synchrotron radiation in the optical band. M 87 is classified as FR I based on its radio morphology. Wilson \& Yang (2002) observed the jet with Chandra on July 29 and 30, 2000 and detected it up to a distance of $\sim 21^{\prime \prime}$ from the core in the X-ray band, which implies that the jet is not as strongly aligned along the line of sight (see also Reynolds et al. 1996) as in the case of blazars.

At radio wavelengths, an impressive jet, which extends up to a few tens of kiloparsecs, can be seen. The central engine is thought to be a supermassive black hole (SMBH) with a mass of $M_{\mathrm{BH}} \sim 3 \times 10^{9} M_{\odot}$ (Macchetto et al. 1997). The scale length is thus $r_{g}=G M_{\mathrm{BH}} / c^{2} \sim 4.5 \times 10^{14} \mathrm{~cm} \sim 1.4 \times 10^{-4} \mathrm{pc}$. Using the Hubble Space Telescope (HST), Biretta et al. (1999) observed superluminal apparent motions of about $4 c-6 c$ beyond $400 \mathrm{pc}$ for the internal knots, between 1994 and 1998, thus confirming that the jet is relativistic. They conclude that the jet is oriented within $19^{\circ}$ of the line of sight.
Due to the presence of an SMBH in the core and the presence of the jet, M 87 was deemed an interesting candidate for $\mathrm{TeV}$ emission. Le Bohec et al. (2004) reported an upper limit observed with Whipple in 2000 and 2001, simultaneously with X-ray flares observed by RXTE. HEGRA observed M 87 in 1998 and 1999 for a total exposure of $77 \mathrm{~h}$ after data quality selection (Aharonian et al. 2003; Beilicke et al. 2004). A 4.1 $\sigma$ significance was recorded and an integrated flux $(E>730 \mathrm{GeV})$ of $3.3 \%$ Crab was measured.

Recently, Aharonian et al. (2006) observed M 87 with the High Energy Stereoscopic System (HESS) ${ }^{1}$ between 2003 and 2006 in $89 \mathrm{~h}$ live-time with a $13 \sigma$ detection and discovered variations on timescales of about 2 days, 10 times faster than observed in any other waveband. This shows that the emission region is very compact, with a dimension on the order of a few Schwarzschild radii. These observations, confirming the detection by HEGRA (Beilicke et al. 2005), are particularly interesting since M 87 is the first non-BL Lac extragalactic object ever observed at $\mathrm{TeV}$ energy. Radio-loud galaxies contain AGNs with jets like blazars, but the jet emission is less strongly boosted due to larger viewing angles between the jet and the observer's

\footnotetext{
${ }^{1}$ http://www .mpi-hd.mpg.de/hfm/HESS/
} 
line of sight. It is therefore a challenge for standard models of $\mathrm{TeV}$ blazars to explain the very high-energy (VHE) emission of M 87.

In this paper, we present a modified synchrotron selfCompton (SSC) scenario to explain the VHE emission of M 87. Classic SSC models (e.g. Gould 1979; Inoue \& Takahara 1996; Bloom \& Marscher 1996; Chiaberge \& Ghisellini 1999; Katarzyński et al. 2001) are applied to blazars, which are beamed sources, and cannot account for the observations of radiogalaxies like M 87. Our goal is to further develop one of these models to reconcile beamed and unbeamed sources in the same framework of models. Such propositions for unification of AGNs have already been studied considering orientation effects (e.g. Antonucci 1993; Urry \& Padovani 1995), or radio/X-ray power among BL Lac objects, flat-spectrum radio-loud quasars (FSRQs) and FR Is (e.g. Fossati et al. 1998; Ghisellini et al. 1998; Capetti et al. 2000).

A short description of the leptonic blob-in-jet model for TeV blazars is found in Sect. 2, and its development and application to M 87 are described in Sects. 3 and 4. In the framework of misaligned BLLac-like objects, we then try to predict VHE fluxes for objects with optical/X-ray extended jets in Sect. 5. The implications for unification schemes of AGNs are discussed in Sect. 6.

\section{2. "Blob-in-jet" leptonic SSC model}

We intend to model the multiwavelength spectrum of M 87 in the framework of a quasi-homogeneous SSC scenario, successfully used to account for the overall emission of blazars, such as Mrk 501 and Mrk 421. Our model relies on the basic scenario presented in Katarzyński et al. (2001, 2003, and references therein), who give the details for the computation of the radiative transfer and emission by SSC processes in a single spherical blob of plasma moving at relativistic speed along the jet axis. The blob, immersed in a uniform magnetic field, is assumed to be located inside the jet, close to the central engine. An inhomogeneous conical extended jet model is also used to explain the emission from radio to ultraviolet wavelengths (see Sect. 2.2 in Katarzyński et al. 2001, for more details). The absorption by the infrared extragalactic background light at VHE is taken into account and modeled using the estimations as described in Stecker et al. (2006) and references therein. Here we model only nearby AGNs, so this effect can be neglected. The blob-in-jet model is particularly well-adapted to the description of blazars, for which the jet is very close to the line of sight. In the following, the assumed cosmology is $H_{0}=70 \mathrm{~km} \mathrm{~s}^{-1} \mathrm{Mpc}^{-1}$ for an Einstein-de Sitter universe, with $\Omega_{\Lambda}=0.7$ and $\Omega_{\mathrm{m}}=0.3$.

We assume that the population of electrons, which is responsible for the non-thermal emission in leptonic models, has a number density that can be described by a broken power law:

$N_{\mathrm{e}}(\gamma)=\left\{\begin{array}{ll}K_{1} \gamma^{-n_{1}} & \gamma_{\min } \leqslant \gamma \leqslant \gamma_{\mathrm{br}} \\ K_{2} \gamma^{-n_{2}} & \gamma_{\mathrm{br}} \leqslant \gamma \leqslant \gamma_{c}\end{array} \quad\left[\mathrm{~cm}^{-3}\right]\right.$

where $K_{2}=K_{1} \gamma_{\mathrm{br}}^{n_{2}-n_{1}}$ and $\gamma=E / m c^{2}$, where $m$ is the electron mass and $E$ its energy. These electrons radiate up to the X-ray range through the synchrotron process, and then re-interact with their own emitted photons by inverse Compton (IC) scattering, which is the so-called synchrotron self-Compton process. This synchrotron emission comes from a population of electrons different from those producing the radio-IR emission of the extended jet.
The SSC model has 8 significant parameters. The macrophysics processes are described by the magnetic field $B$, the radius of the emitting blob $r_{\mathrm{b}}$ and the Doppler factor $\delta_{\mathrm{b}}=$ $\left[\Gamma_{\mathrm{b}}\left(1-\beta_{\mathrm{b}} \cos \theta\right)\right]^{-1}$, where $\beta_{\mathrm{b}}$ is the speed of the moving blob in $c$ unit, $\Gamma_{\mathrm{b}}=\left(1-\beta_{\mathrm{b}}^{2}\right)^{-1 / 2}$ is the blob Lorentz factor, and $\theta$ the viewing angle. The radiative processes are parametrized by the description of the population of emitting particles, with the parameters $K_{1}, \gamma_{\mathrm{br}}, \gamma_{c}, n_{1}$, and $n_{2}$ from Eq. (1). The value of $\gamma_{\min }$ is not crucial for interpreting the spectral energy distribution (SED), nor is $\gamma_{c}$, although it can become very relevant in cases where the X-rays have a hard slope with a spectral differential index $\alpha<1$ (in the common $f_{v} \propto v^{-\alpha}$ notation). All these parameters can be constrained when detailed spectral data are available for a wide frequency range.

In the present case, the spectral coverage of the nucleus of M 87 is sparse, so we need to find other ways to constrain the parameters. One important constraint comes from the variability:

$r_{\mathrm{b}}<\frac{c \delta_{\mathrm{b}}}{1+z} \Delta t_{\mathrm{obs}}$

where $\Delta t_{\mathrm{obs}}$ is the variability timescale in the observer frame, implying $r_{\mathrm{b}} / \delta_{\mathrm{b}} \lesssim 5 \times 10^{15} \mathrm{~cm}$ for M 87 .

The region of emission is then assumed to be close to the $\mathrm{SMBH}$, to fulfill the variability constraint within magnetohydrodynamic (MHD) jet models. For instance, McKinney (2006) models the jet formation zone using general relativistic MHD simulations, applicable to GRBs, AGNs as M 87, and black hole X-ray binaries. He describes the broadening zone of the jet in the vicinity of the central black hole, and finds the Alfvén surface at $\sim 50 r_{g}$. We assume that the emission zone is located slightly above this surface to allow shocks and Fermi acceleration processes to develop in the jet. The results of McKinney (2006) further constrain some of our parameters for a distance of $\sim 100 r_{g}$ from the SMBH, such as the value of the Lorentz factor $\Gamma_{\mathrm{b}} \lesssim 10$ of the plasma blobs, the magnetic field $B$, and the half-opening angle $\varphi(r)$ of the jet given by his Eq. (24).

The case of M 87 is of particular interest since its jet is exceptionally well mapped in radio VLBI. Biretta et al. (2002) observed the core of M87 in VLBI in February 1995 and March 1999, and showed that the opening angle increases quickly with decreasing distance to the core region, on the $0.01 \mathrm{pc}\left(\sim 70 r_{g}\right)$ scale. This kind of widening at the base of the jet has also been observed in Cen A on the $0.1 \mathrm{pc}\left(\sim 19000 r_{g}\right)$ scale by Horiuchi et al. (2006) using the VLBI Space Observatory Program. A broadening zone in the jet formation region is also found in MHD simulations. Moreover, the recently detected short-term $\mathrm{TeV}$ variability seems to exclude the extended and outer regions as the source for VHE emission in M 87. This is also argued by Georganopoulos et al. (2005), who present a modified leptonic model applied to M 87 that takes into account a deceleration of the inner flow along the base of the jet. It thus appears quite natural to assume that the VHE $\gamma$-rays are emitted in the core-widened jet region.

We can then imagine that there are blobs of plasma, harboring very high-energy electrons and propagating in the widened jet formation zone, which are dragged along with the bulk jet outflow. In the case of misaligned objects such as M 87, this can easily result in one blob moving along the line of sight and thus having about the same Lorentz factor as for blazars, allowing reproduction of the $\mathrm{TeV}$ emission in the framework of classic SSC models. However a model with a single relativistic blob moving and emitting exactly towards the observer would be statistically unlikely. 


\section{Multi-blob model}

A way of dealing with this statistical issue is to assume that the emission zone is a spherical cap centered on the SMBH, limited by the sheath of the jet, and filled with several similar homogeneous blobs. Consequently, as mentioned in Aharonian et al. (2006), we can consider differential Doppler-boosting in the jet formation zone, near the core region.

This cap is located at a given distance $R_{\text {cap }}$ from the SMBH, which is a new free parameter in our model. However, $R_{\text {cap }}$ can be constrained by MHD simulations (e.g. McKinney 2006) if we assume that it is located slightly above the Alfvén surface, which is at about $50-100 r_{g}$ from the SMBH. This surface is continuous, but does not need to be homogeneous. We model it with a pattern of several blobs, whose individual radii are typically smaller than in the case of the "blob-in-jet" scenario.

For the sake of simplicity, we assume the presence of 7 blobs in this zone, one central blob and 6 further blobs distributed on a hexagon, located at $100 r_{g}$ from the SMBH, with macroscopic parameters derived from McKinney (2006) as specified in Sect. 2. This choice for the number of blobs is justified by the fact that the resulting diameter of the cap is in the same order of magnitude as the characteristic size of the emitting zone from previous studies.

The smaller the radius of the individual blob, the more our model resembles a continuous zone model. The choice of discrete adjacent blobs leads to two extreme situations:

- "Inter-blob" case: the line of sight passes exactly through the gap between three blobs;

- "On-blob" case: the line of sight is exactly aligned with the velocity vector of the central blob, called No. 0.

The Doppler factor for each blob No. $i$ (where $i \in \llbracket 0 ; 6 \rrbracket)$ is now defined as:

$\delta_{\mathrm{b}}^{i}=\frac{1}{\Gamma_{\mathrm{b}}\left(1-\beta_{\mathrm{b}} \cos \alpha_{i}\right)}$

where $\alpha_{i}$ is the angle between the velocity vector of blob No. $i$ and the line of sight. If the line of sight is between three blobs ("inter-blob" case), then these blobs have the same Doppler factor and their contribution to the total flux is equal, while the 4 other blobs have contributions to the total flux that decrease with increasing blob radius $r_{\mathrm{b}}$. If the line of sight is aligned with the velocity vector of the central blob ("on-blob" case), then the highest Doppler factor is $\delta_{\mathrm{b}}^{0}$. In that case the six other blobs all have the same Doppler factor; and although smaller than $\delta_{\mathrm{b}}^{0}$, their individual contributions are not negligible in the total observed flux, especially if the seven blobs are all moving along in the same direction ${ }^{2}$. It should be noted that some models involve acceleration as the jet is collimated (e.g. Melia et al. 2002; Vlahakis \& Königl 2004), which is on the parsec scale. In such models, a gradient in flow velocity across the width of the jet can also be present, but is usually small compared to the radial velocity profile. Therefore we choose here to neglect this transverse gradient, which is of second order for our purpose, and we assume that all the blobs have the same Lorentz factor, although they are ejected at slightly different angles.

Figure 1 describes the geometry of our model in the "onblob" case. The central blob is moving along the line of sight, and the six other blobs are each moving along a direction slightly different from the blob $\mathrm{n}^{\circ} 0$ by an angle $\mathrm{d} \alpha$. The angle $\mathrm{d} \alpha$ is given by $\mathrm{d} \alpha=2 \arcsin \left(r_{\mathrm{b}} / R_{\text {cap }}\right)$. The viewing angle $\theta$ is defined as the

\footnotetext{
${ }^{2}$ which is the case for $r_{\mathrm{b}} \leqslant r_{g}$.
}

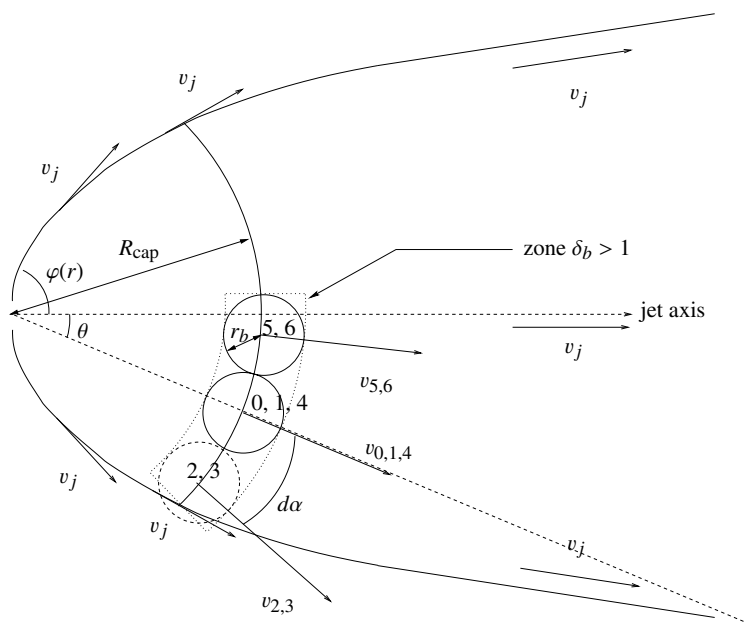

Fig. 1. Geometric side view of the jet formation zone ("on-blob" case). $v_{j}$ represents the velocity of the jet, the blobs are numbered from 0 to 6 , $v_{0 \rightarrow 6}$ are the velocities of the blobs, $\theta$ is the viewing angle with respect to the jet axis, $\varphi(r)$ is the opening angle, $r_{\mathrm{b}}$ is the radius of an individual blob, and $R_{\text {cap }}$ is the distance of the blobs from the SMBH. In this sketch, the contributions to the total flux of the blobs No. 2 and 3 would be rejected, since they lie outside the jet.

angle between the line of sight and the jet axis for this multi-blob model. The individual radius $r_{\mathrm{b}}$ assumed equal for all blobs is a free parameter of our model. Depending on the observed angle $\theta$, it can happen that in the simulation a blob moves outside the jet and is therefore neglected.

We can then compute the radiative transfer of each blob in its own source frame, as explained in Katarzyński et al. (2001). For each blob, the seed photons for the inverse Compton scattering are those generated within the blob from synchrotron radiation. The total flux in the observer frame is the sum of the contribution of each blob. We neglect the contribution of the eventual blobs for which $\delta_{\mathrm{b}}<1$, which is possible if $\alpha_{i}>$ $\arccos \left[\left(\Gamma_{\mathrm{b}}-1\right) /\left(\Gamma_{\mathrm{b}} \beta_{\mathrm{b}}\right)\right]$.

Figure 2 shows the SED of the contribution from the single central blob in the "on-blob" case, as well as the contribution of the six adjacent blobs. Adjacent blobs have the same Doppler factor, so they all contribute equally to the SED. It also shows the sum of all the contributions in the "inter-blob" case, where the line of sight is exactly between three blobs. If $r_{\mathrm{b}}$ is sufficiently large, the closest blob to the line of sight in the "on-blob" case completely dominates the apparent flux. This can be the case in almost all the situations we study here.

\section{Application to M 87}

\subsection{The observed SED}

To construct the SED of the core jet, we carefully select the following data from the literature. We used $\gamma$-ray observations of 2004 and 2005 by HESS (Aharonian et al. 2006), representing lower and higher states respectively in the next figures. The Whipple upper limit at $400 \mathrm{GeV}$ observed between 2000 and 2003 is taken from Le Bohec et al. (2004). The HEGRA point at $730 \mathrm{GeV}$ obtained in 1998 and 1999 is taken from Beilicke et al. (2004).

The Chandra data from the nucleus region obtained on April 20 and July 30, 2000 were taken from Perlman \& Wilson (2005) who conclude that the nuclear X-ray emission originates 


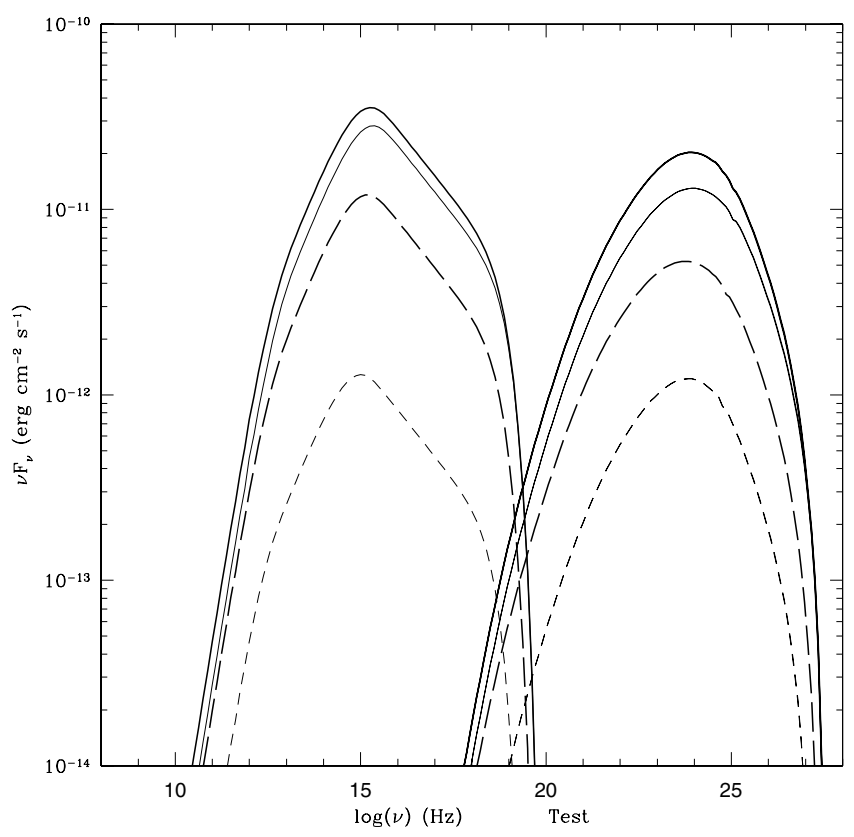

Fig. 2. Illustrative example of the SED of the multi-blob model. The thin solid line shows the contribution of the central blob, the most strongly beamed blob. The thin dashed line shows the contributions of each of the six other blobs, and the bold solid line shows the sum of the contributions of all the blobs in the "on-blob" case. The bold dashed line shows the sum of all the contributions in the "inter-blob" case. The closest blob to the line of sight (thin solid line) is overwhelmingly dominant for high enough values of $r_{\mathrm{b}}$.

from the jet and could extract a 1 arcsec nucleus spectrum, thus excluding HST-1. The XMM-Newton data taken on June 19, 2000 were found in Böhringer et al. (2001). It should be noted that the XMM-Newton data have the same spectral slope, but a higher flux density than the Chandra data, and they do include $H S T$-1 within a $4^{\prime \prime}$ extraction region. Perlman et al. (2001) provide data taken by the Gemini North telescope at 10 microns in May 2001. The observations by the HST in the optical and UV bands in 1991 are reported in Sparks et al. (1996). The observations of the core of M 87 by the VLA in the radio band and by the Palomar observatory in the optical band between 1979 and 1985 were found in Biretta et al. (1991).

We also took upper limits in $\gamma$-ray by EGRET between 1991 and 1993 into account (Sreekumar et al. 1994), and in UV by $E U V E$ (Berghöfer et al. 2000). All the other data were taken from the NASA/IPAC Extragalactic Database (NED).

Although we do not have simultaneous data, the Chandra data of 2000 and the HESS $\gamma$-ray data of 2004 both correspond to low states of activity in their own spectral range. We therefore associate them, assuming that they are representative of a typically low state. Indeed, regarding the X-ray data, the Chandra observations of 2000 also correspond to the lower state of activity recently published (Perlman et al. 2003). For the models, we choose to take the mean spectral slope of the Chandra data into account. The radio to optical/UV data are also not simultaneous with the $\gamma$-ray data, but this is not problematic since the radio contribution is thought to come from the extended jet, with different characteristics from the VHE emitting zone.

\subsection{The SSC models}

In the case of M 87, the observation angle $\theta$ between the jet axis and the line of sight is at most $19^{\circ}$ (Biretta et al. 1999). The

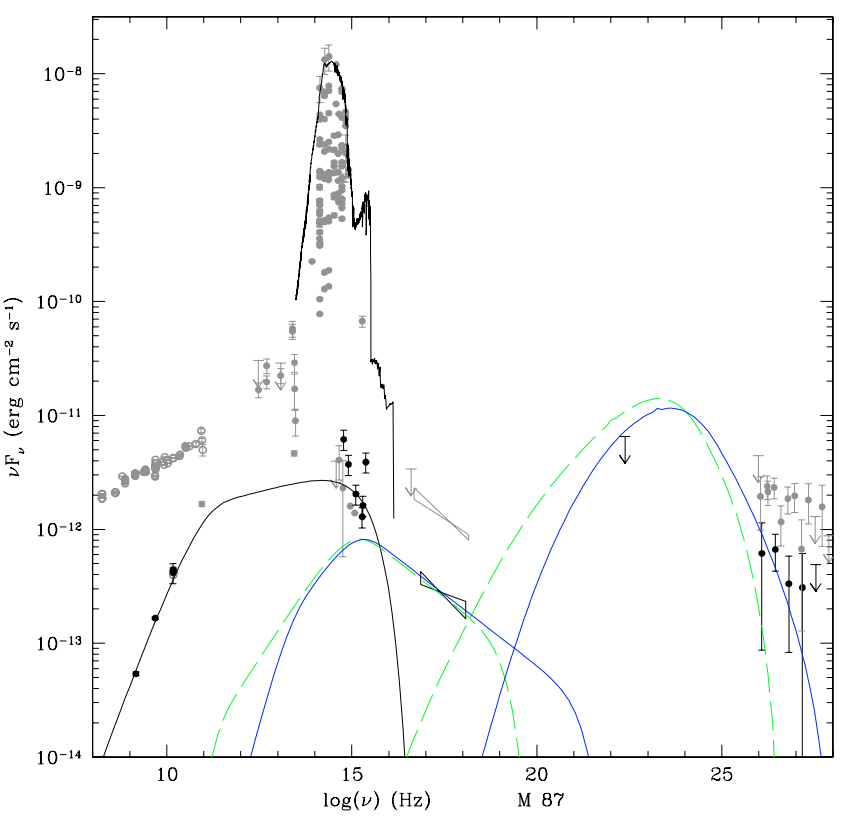

Fig. 3. Tentative modeling of the SED of M 87 within a standard blobin-jet scenario, with $\delta_{\mathrm{b}}=3.07$ presented by the dashed green line. The solid blue line shows the SED of M 87 emitted by a single blob moving along the line of sight in the jet formation region, with $\delta_{\mathrm{b}}=8$, which can describe the data. The data points that bring direct constraints to our model are shown in black, the other less constraining points are represented in gray. The black line in the optical band shows the host galaxy, assumed to be elliptic. This contribution was computed using results from the code PEGASE (Fioc \& Rocca-Volmerange 1997). The radio data shown by gray empty circles, obtained from the NED and with fluxes $\sim 10^{-12} \mathrm{erg} \mathrm{cm}^{-2} \mathrm{~s}^{-1}$, come from the extended kiloparsecscale jet and radio lobes. The black line from radio to UV/X represents a model of the extended inner jet (see Katarzyński et al. 2001, 2003), with the corresponding radio data with fluxes $\sim 10^{-13} \mathrm{erg} \mathrm{cm}^{-2} \mathrm{~s}^{-1}$ reported in black. The bump peaking at $\sim 10^{15} \mathrm{~Hz}$ is due to synchrotron emission and the bump peaking at $\sim 10^{23} \mathrm{~Hz}$ is due to inverse Compton, both from the VHE zone (see Cols. 1 and 2 of Table 1 for the corresponding parameters). (See the electronic edition of the Journal for a color version of this figure).

blob-in-jet model cannot describe the VHE emission of the source correctly, as it would require very high Doppler factor, which is not sustainable. This is illustrated in Fig. 3. We can obviously see that this model, for which we present the best solution for the SED of M 87 within the blob-in-jet scenario described in Sect. 2 with $\delta_{\mathrm{b}}=3.07$ assuming $\theta=19^{\circ}$ (see Col. 1 in Table 1 for the corresponding parameters), cannot account for the VHE emission. Higher values of $\delta_{\mathrm{b}}$ are not allowed here because of the high value of $\theta$. The well-defined X-ray slope deduced from the observations strongly constrains the second index $n_{2}$ in the electron energy distribution (see Eq. (1)) and significantly reduces the parameter space.

Figure 3 presents the resulting SED emitted when one consider a single blob moving along the line of sight in the jet formation zone with $\delta_{\mathrm{b}}=8$. The corresponding parameters can be found in Col. 2 of Table 1, where $\theta$ is defined as the angle between the line of sight and the velocity vector of the single blob. Obviously this model describes the observations much better. However, as pointed out in Sect. 2, it is based on an ad hoc assumption. Moreover, it is difficult to "keep" the generated IC bump below the EGRET upper limit, although we assumed a low state for the activity of the AGN. 
Table 1. Parameters used in the different generated SEDs.

\begin{tabular}{|c|c|c|c|c|c|c|c|c|}
\hline Model & "Blob-in-jet & Single blob & \multicolumn{6}{|c|}{ Multi-blob } \\
\hline $\begin{array}{l}\text { Object } \\
\text { figure }\end{array}$ & $\begin{array}{c}\text { M } 87 \\
3 \text { (green) }\end{array}$ & $\begin{array}{c}\text { M } 87 \\
3 \text { (blue) }\end{array}$ & $\begin{array}{c}\mathrm{M} 87 \\
4\end{array}$ & $\begin{array}{c}\text { M } 87 \\
5\end{array}$ & $\begin{array}{c}3 \mathrm{C} 273 \\
6\end{array}$ & $\begin{array}{c}\text { Cen A } \\
7 \text { (blue) }\end{array}$ & $\begin{array}{c}\text { Cen A } \\
7 \text { (green })\end{array}$ & $\begin{array}{c}\text { PKS 0521-36 } \\
8\end{array}$ \\
\hline$\overline{\delta_{\mathrm{b}}}$ & 3.07 & 8 & - & - & - & - & - & - \\
\hline$\Gamma_{\mathrm{b}}$ & - & - & 4.1 & 10.0 & 7.4 & 8.14 & 20.0 & 1.5 \\
\hline$\theta$ & $19^{\circ}$ & $1^{\circ}$ & $15^{\circ}$ & $15^{\circ}$ & $15^{\circ}$ & $25^{\circ}$ & $25^{\circ}$ & $25^{\circ}$ \\
\hline$R_{\text {cap }}\left[r_{q}\right]$ & - & - & 100.0 & 100.0 & 100.0 & 100.0 & 100.0 & 100.0 \\
\hline$B[\mathrm{G}]$ & 1.0 & 0.5 & 0.5 & 0.01 & 3.0 & 2.0 & 10.0 & 1.0 \\
\hline$r_{\mathrm{b}}[\mathrm{cm}]$ & $1.2 \times 10^{14}$ & $4.0 \times 10^{13}$ & $1.5 \times 10^{13}$ & $2.8 \times 10^{14}$ & $2.0 \times 10^{15}$ & $1.0 \times 10^{14}$ & $8.0 \times 10^{13}$ & $9.0 \times 10^{14}$ \\
\hline$K_{1}\left[\mathrm{~cm}^{-3}\right]$ & $1.5 \times 10^{7}$ & $3.5 \times 10^{7}$ & $7.7 \times 10^{7}$ & $1.8 \times 10^{4}$ & $1.8 \times 10^{6}$ & $9.0 \times 10^{7}$ & $4.0 \times 10^{4}$ & $3.0 \times 10^{6}$ \\
\hline$n_{1}$ & 2.0 & 2.0 & 2.0 & 1.5 & 2.0 & 2.0 & 2.0 & 2.0 \\
\hline$n_{2}$ & 3.5 & 3.5 & 3.5 & 3.5 & 4.1 & 3.0 & 3.5 & 2.5 \\
\hline$\gamma_{\min }$ & $10^{2}$ & $10^{3}$ & $10^{3}$ & $10^{3}$ & 1 & $3.0 \times 10^{2}$ & $10^{3}$ & $10^{3}$ \\
\hline$\gamma_{\mathrm{br}}$ & $10^{4}$ & $10^{4}$ & $10^{4}$ & $10^{4}$ & $1.6 \times 10^{3}$ & $5.0 \times 10^{2}$ & $3.5 \times 10^{5}$ & $5.0 \times 10^{4}$ \\
\hline$\gamma_{c}$ & $10^{6}$ & $10^{7}$ & $10^{7}$ & $10^{7}$ & $10^{6}$ & $4.0 \times 10^{3}$ & $6.0 \times 10^{6}$ & $10^{6}$ \\
\hline
\end{tabular}

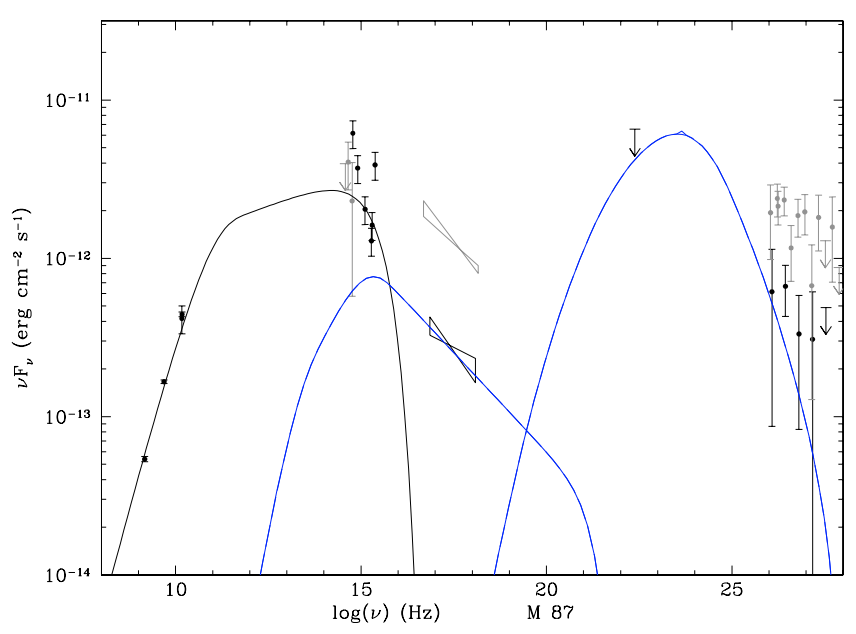

Fig. 4. SED of M 87 within the multi-blob scenario for small blob radius $\left(r_{\mathrm{b}}=1.5 \times 10^{13} \mathrm{~cm}\right.$, see Col. 3 in Table 1$)$. The two extreme "on-blob" and "inter-blob" cases are identical here because of the low value of $r_{\mathrm{b}}$. (See the electronic edition of the Journal for a color version of this figure).

Throughout this paper, our results are not fits to the data, but rather solutions of models that are meant to describe the data best. Our purpose is to figure out whether our model can correctly describe the current available data for different objects. We did not intend to fine-tune the parameters of our model but to sort orders of magnitude out for these parameters.

One SED of M 87 generated within the multi-blob model is presented in Fig. 4, with parameters very similar to the single blob model of Fig. 3 (see Col. 3 in Table 1). Since the former is a generalization of the latter, the resulting spectrum is rather similar, as one would expect. In this case, the value of the individual blob radius $r_{\mathrm{b}}$ is so low that all the blobs are moving close to the line of sight. The "on-blob" and the "inter-blob" cases give the same contribution to the SED and are overlaid in Fig. 4.

The blob radius is rather small in this case, resulting in a VHE-emitting zone smaller than the Schwarzschild radius. It should be noted that small features compared to the Schwarzschild radius, possibly responsible for the VHE emission, can develop beyond the Alfvén surface due to turbulence or reconfined shocks, but this issue is beyond the scope of this work.
It is commonly believed, however, that the size of the VHE emitting zone cannot be much smaller than the Schwarzschild radius, which is a natural scale for the processes in the vicinity of the SMBH. Moreover, the emitting zone must be large enough to allow the acceleration of particles to develop. Very small blobs may disappear rapidly, in $\sim 10$ min due to adiabatic expansion, which is especially important in the broadened zone of the jet; however, a long, stable emission is possible, even from small blobs. The emitting zone can be located at a stable stationary shock front, above the Alfvén surface. It initiates the acceleration and thus the radiation of particles of a large number of small blobs continuously crossing the shock, thus providing a quiescent background of VHE emission. Density fluctuations in the injection of material could then generate flares as seen at VHE. In fact, the only problem with small blobs is that in this case the paving of the jet is not complete because of the discretization applied in our code.

In order to be more conservative and to fulfill the constraint $r_{\mathrm{b}} \gtrsim r_{g}$, we analyzed another possibility with a low magnetic field. It is presented in Fig. 5 with associated parameters in Col. 4 of Table 1. In that case, the result predicted by MHD models with a strong magnetic field in the vicinity of the central engine is not strictly fulfilled; however, a local decrease in the magnetic field can be achieved by a simple expansion of the emitting zone. This solution may appear preferable for conservative reasons to the size of the emitting zone.

A satisfactory solution for the high state observed by HESS in 2005 is also possible in this case within the multi-blob scenario as shown in Fig. 5. Interestingly, we predict a radical change in the X-ray regime. Clearly the parameters are not wellconstrained here due to the lack of any simultaneous data, especially in the X-rays; however, this does illustrate the capability of the multi-blob model to generate spectra that are sufficiently hard in the TeV range to reproduce the most recent HESS data. A slight modification of standard SSC models for TeV blazars therefore appears successful and can account for all VHE data on the radiogalaxy M 87 that are available up to now.

\section{Predictions for other radiogalaxies with optical/X-ray extended jets}

We now apply the SSC multi-blob model to different sources that have the peculiarity of showing an extended optical or X-ray jet, which suggests at least a moderate beaming towards the observer as in the case of M 87. This allows comparison of the 


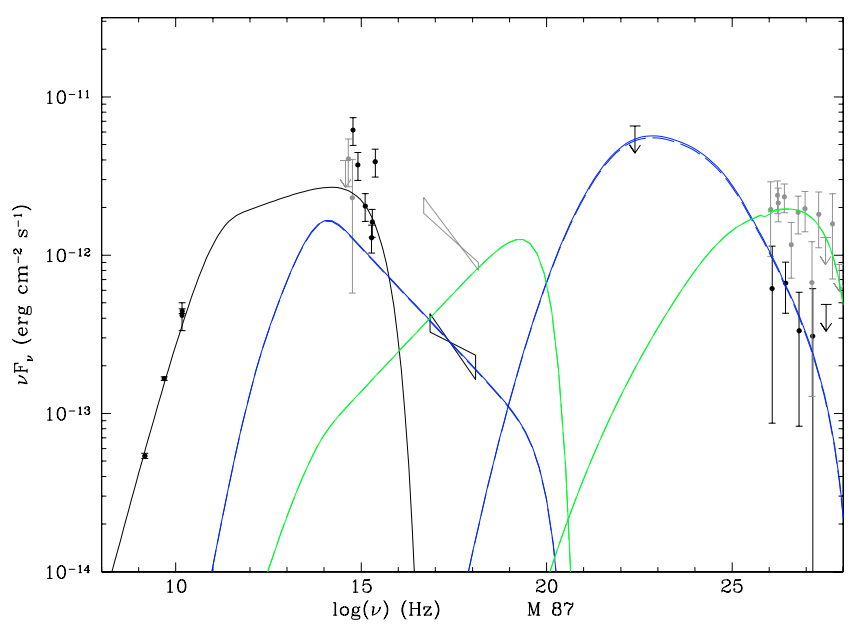

Fig. 5. SED of M 87 within the multi-blob scenario, assuming a low magnetic field ( $B=10 \mathrm{mG}$, see Col. 4 in Table 1) in blue lines. The solid lines show the "on-blob" case, while the dashed lines represent the "inter-blob" case. The green lines show a solution for the high state observed by HESS in 2005, with the same parameters as for the observations of 2004, except for $r_{\mathrm{b}}=8.0 \times 10^{13} \mathrm{~cm}, K_{1}=2.2 \times 10^{4} \mathrm{~cm}^{-3}$ and $n_{2}=2.5$. (See the electronic edition of the Journal for a color version of this figure).

multi-blob scenario to other types of AGNs and a prediction of whether these sources can be detectable at VHE or not by present Čerenkov arrays. We chose three AGNs not belonging to the genuine blazar class, presented here with increasing viewing angles, such that their fluxes are boosted by relativistic effects less and less. We stress that not all the data presented here are simultaneous and that the sources undergo strong variations.

\section{1. $3 C 273$}

3C $273(z=0.158$, Strauss et al. 1992) is the first quasar that was identified as a high-redshift object (Schmidt 1963) and the beststudied one. It hosts an SMBH whose mass is at least $\sim 2.0 \times$ $10^{9} M_{\odot}$ as inferred from studies of Balmer lines (Paltani \& Türler 2005) and its maximum acceptable viewing angle is about $15^{\circ}$ (Unwin et al. 1985).

Blazars display featureless X-ray contribution, but radiogalaxies can have a much more complex environment at low energies (e.g. Grandi et al. 2006). However, since the purpose here is to model the non-thermal contributions of these objects, we decided to consider the X-ray contribution as dominated by the jet emission, keeping only a feature in soft X-ray as a signature of the accretion disk (Grandi \& Palumbo 2007).

Since this source is highly variable, one needs to be careful to select simultaneous data. The X-ray data presented here are BeppoSAX observations ${ }^{3}$ taken from Giommi et al. (2002). We report in Fig. 6 the upper limit at $3 \sigma$ obtained by HESS in 2005 (Aharonian et al. 2005). All the other data points were taken from Türler et al. (1999) who report an average spectrum compiled from 30 years of observations. Observations by BeppoSAX on January 13, 15, 17, and 22, 1997 lie within the observation period of $C G R O$ taken by COMPTEL and EGRET between December 10, 1996 and January 28, 1997 (Collmar et al. 2000), which are also reported in Türler et al. (1999). We thus have simultaneous data for the $\mathrm{X} / \gamma$-ray bump in one of the highest states, which puts an important constraint on the models.

\footnotetext{
${ }^{3}$ See http://www.asdc.asi.it/blazars/
}

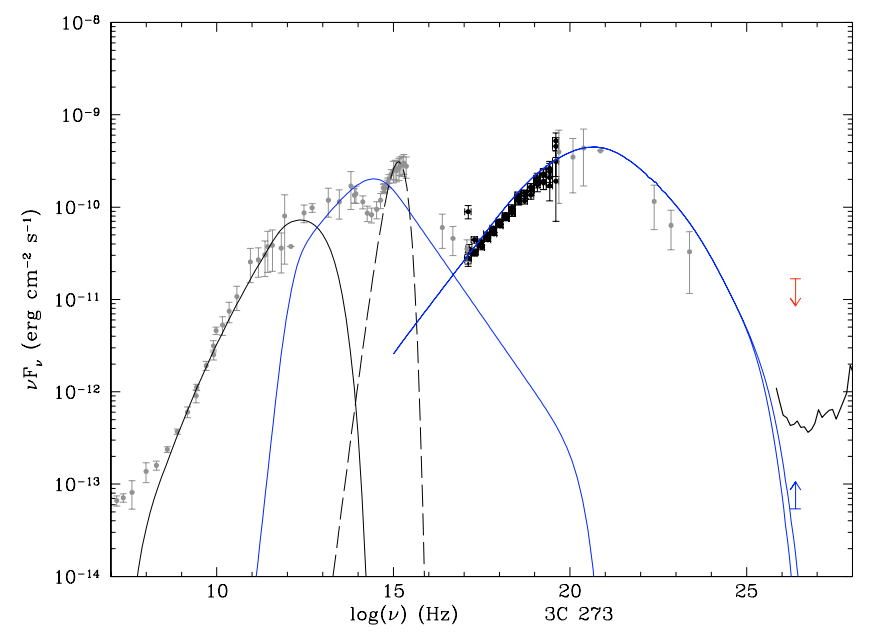

Fig. 6. SED of 3C 273 with anticipated VHE flux. The solid blue line shows the average of the "on-blob" and "inter-blob" cases (see Col. 5 in Table 1 for the corresponding parameters). The dashed line represents a simple blackbody model to illustrate the contribution of the big blue bump component. The upper limit obtained in 2005 with the HESS telescope array is shown in red. The blue lower limit shows the expected CTA sensitivity in $50 \mathrm{~h}$ of observation. (See the electronic edition of the Journal for a color version of this figure).

The nature of the X-ray emission of 3C 273 is still an unresolved issue. The high-frequency bump, thought to be probably due to IC emission, would present a peak at a rather low frequency compared to other AGNs. This implies that either 3C 273 does not emit at detectable VHE levels or that the nature of this bump is synchrotron, implying then the presence of a hypothetical IC bump at ultra high energies.

Assuming that the hard X-ray emission is indeed due to inverse Compton emission, Fig. 6 shows the SED of 3C 273 with the predicted VHE flux (see Col. 5 of Table 1 for the corresponding parameters). Modeling the SED of 3C 273, we note that changing the value of $\gamma_{\text {min }}$ dramatically affects the X-ray flux by increasing it with decreasing $\gamma_{\min }$, and hence could explain some X-ray flares, as also suggested by Georganopoulos et al. (2006) in the case of external inverse Compton emission on the cosmic microwave background. The value of $\gamma_{\min }$ is strongly constrained here by the precise shape of the X-ray spectrum. In the following figures, the V-shaped curve at VHE $\left(\sim 10^{26} \mathrm{~Hz}\right)$ shows the HESS sensitivity limit for a detection of $5 \sigma$ in $50 \mathrm{~h}$ of observing a source at a mean zenith angle of $30^{\circ}$. The expected sensitivity of the next generation CTA project of $\sim 0.1 \%$ Crab flux at $1 \mathrm{TeV}$ for $50 \mathrm{~h}$ of observation is shown as a blue lower limit.

Since the results for the "on-blob" and "inter-blob" scenarii are not very different in this case, we show the average of the two. We should stress that low-frequency data are not simultaneous with the $\mathrm{X} / \gamma$-ray data that we selected. The modeled synchrotron bump has a higher flux density than the optical data because we are dealing with a high state of activity in $\gamma$-rays as reported by Collmar et al. (2000). We only predict a very marginal detection of 3C 273 by HESS in its low-energy range, depending on the energy threshold (but see also Georganopoulos et al. 2006). Furthermore it should be recalled that Collmar et al. (2000) report an active state in $\gamma$-rays and Türler et al. (2006) a high level of the non-thermal emission at the epoch of the data we are considering. Even in a high state, we do not expect a strong level of VHE $\gamma$-ray within our scenario. 
A strong detection of 3C 273 at VHE with the current generation of Čerenkov arrays would be difficult to explain within our SSC nuclear scenario. A possibility would be to invoke disparity among the various emitting plasma blobs. Our model shows the presence of a well-defined inverse Compton bump; different magnetic fields or electron energy distributions among the blobs could result in a tail of the IC bump at VHE that could account for a VHE detection. An alternative would be an extended emission due to external inverse Compton radiation, which is then expected to be not very variable. In all cases, further observations with GLAST (10 keV-300 GeV) and HESS II, which will extend the spectral domain of HESS I down to $\sim 20 \mathrm{GeV}$ with better sensitivity, are required to disentangle the different plausible scenarii.

\section{2. $\operatorname{Cen} A$}

Cen $\mathrm{A}$ is the nearest radiogalaxy $(z=0.0018$, Graham 1978) and one of the best-studied. The presence of an AGN in Cen A is evident from the radio band to the $\gamma$-rays. Observations from CGRO (Kinzer et al. 1995) show a bump that seems to peak at $\sim 200 \mathrm{keV}$, as pointed out by Steinle et al. (1998). We thus have a definite constraint for the parameters of our model, particularly with regard to describing the population of electrons. As in the case of $3 \mathrm{C} 273$, the IC bump peaks at rather low energy, leading Chiaberge et al. (2001) to note that Cen A could be a misaligned low-energy peaked BL Lac (LBL) object. The value of the viewing angle of the jet is still a controversial issue. For instance, Tingay et al. (1998) claim $\theta \sim 50^{\circ}-80^{\circ}$ for the parsecscale jet, whereas Hardcastle et al. (2003) find $\theta \sim 15^{\circ}$ for the $100 \mathrm{pc}$ scale jet. We choose here to take an intermediate value of $\theta \sim 25^{\circ}$ (See Horiuchi et al. 2006, for a discussion about the different values for the viewing angle of Cen $\mathrm{A}$ found in the literature). The SMBH mass inferred from gas kinematical analysis using a [S III] line is $\sim 1.1 \times 10^{8} M_{\odot}$ (Marconi et al. 2006), but see also Häring-Neumayer et al. (2006), who give $M_{\mathrm{BH}} \sim 6 \times$ $10^{7} M_{\odot}$ using an [Fe II] line.

The data sample chosen here is almost the same as in Chiaberge et al. (2001). The data were retrieved carefully to only take the nucleus into account. The strongly constraining $C G R O / C O M P T E L ~ \gamma$-ray data were taken from Steinle et al. (1998); Rothschild et al. (2006) provided RXTE and INTEGRAL data; HST/NICMOS and WFPC2 data, which we carefully dereddened, are from Marconi et al. (2000) ${ }^{4}$; SCUBA data at $800 \mu \mathrm{m}$ were taken from Hawarden et al. (1993); ISO and SCUBA $(450 \mu \mathrm{m}$ and $850 \mu \mathrm{m})$ data are from Mirabel et al. (1999); VLA data are from Burns et al. (1983). Evans et al. (2004) report X-ray observations by XMM-Newton on February 2 and 6, 2001 and by Chandra on May 9 and 21, 2001 with a photon index $\Gamma=2$ for the parsec-scale jet component. Data from the NED are shown as non-constraining points for comparison. The HESS upper limit based on observations in 2004 with a live-time exposure of $4.2 \mathrm{~h}$ is reported (Aharonian et al. 2005).

We should also point out that, as Cen A harbors a strongly absorbing dust lane and because this source is extremely close and well-resolved, the X-ray data should then be taken only as upper limits. The contribution of the nuclear jet might be contaminated by the accretion disk of the AGN and by the X-ray binaries that are resolved in this object. In this case we would only

4 The reddening correction was applied using the factors carried out by Marconi et al. (2000) themselves.

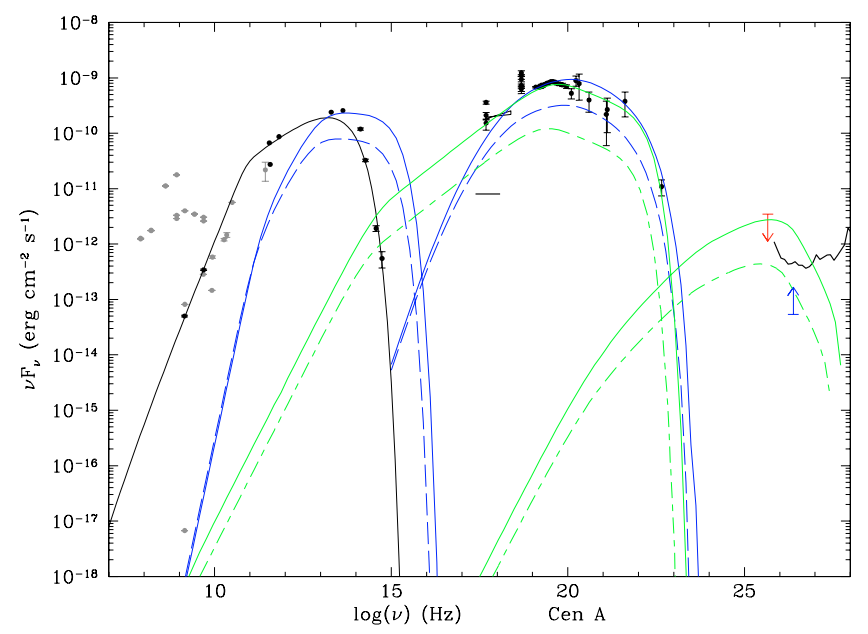

Fig. 7. SED of Cen A within the multi-blob scenario. Solid lines show the "on-blob" emission; the "inter-blob" cases are represented by dashed lines. In blue we show a model assuming that the soft $\gamma$-ray data are inverse Compton emission, while in green we assume a synchrotron emission to describe them (see Cols. 6 and 7 respectively in Table 1 for the corresponding parameters). (See the electronic edition of the Journal for a color version of this figure).

have poor constraints on the emission process. We assume here that all our selected high-energy data come from SSC processes.

Figure 7 shows the SED of Cen A applying the multi-blob model in two cases, $(i)$ assuming that the $\gamma$-ray peak observed by $C G R O$ is inverse Compton radiation (solid and dashed blue lines) or (ii) assuming it to be synchrotron (solid and short dashed-long dashed green lines).

We should also point out that the previous study by Steinle et al. (1998) reports a variability in soft $\gamma$-ray of about 10 days, implying $r_{\mathrm{b}}<2 \times 10^{17} \mathrm{~cm}$ (see Eq. (2)), which is satisfied by our parameters.

Given the results of our model in the first scenario with an IC bump in soft $\gamma$-rays (blue lines), the SSC emission of the central region would definitely not provide a flux high enough to be detectable at VHE (see Col. 6 in Table 1), at least for an SSC emission dominated by the nucleus. This holds even in the case of huge variations in the nuclear emission. This conclusion concurs with Stawarz et al. (2003), who do not expect SSC emission by the nucleus or by the base of the jet of Cen A, but do expect VHE emission that could be detectable by current imaging atmospheric Čerenkov telescopes facilities in the case of an external inverse Compton-emission process on the host-galaxy photon field. For Stawarz et al. (2006), many Fanaroff-Riley type I (FR I) radiogalaxies like Cen A could be TeV sources for which the weak nuclear $\gamma$-ray emission would be absorbed and re-processed by inverse Compton on the starlight photon field, thus generating an isotropic $\gamma$-ray halo. In our model, the lack of simultaneous data prevents us from further constraining the synchrotron bump, which here has a higher flux density than the selected data since we are considering a state of high $\gamma$-ray activity.

In the solid ("on-blob" case) and short dashed-long dashed ("inter-blob" case) green lines in Fig. 7, we present an SED of Cen A assuming now that the soft $\gamma$-ray peak is of synchrotron origin. In this case (see Col. 7 in Table 1), we expect detection of the core of Cen A at VHE by the HESS telescope array within $50 \mathrm{~h}$ of observation. It should be noted that Bai \& Lee (2001) also predict the synchrotron bump to be in the soft $\gamma$-ray range 


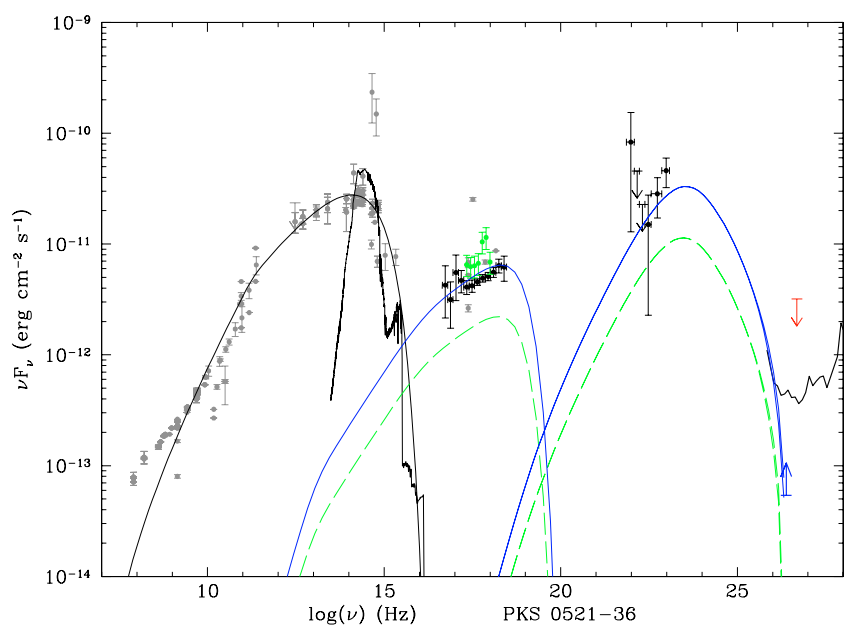

Fig. 8. SED of PKS 0521-36 with anticipated VHE flux modeled in the multi-blob framework. The solid blue line is the "on-blob" case, while the dashed green line shows the "inter-blob" emission (see Col. 8 in Table 1 for the corresponding parameters). The CANGAROO upper limit is reported in red. (See the electronic edition of the Journal for a color version of this figure).

and the inverse Compton bump to peak around $1 \mathrm{TeV}$ in the context of SSC models, which supports our latter model.

\subsection{PKS 0521-36}

PKS 0521-36 is an FSRQ object with an optical jet located at $z=0.055$ (Keel 1985). The central SMBH has a mass of $\sim 3.3 \times$ $10^{8} M_{\odot}$ (Woo et al. 2005). Pian et al. (1996), and more recently Tingay \& Edwards (2002) mention the absence of superluminal motions in its jet, in contrast to the case of 3C 273, implying that the beaming effect is much weaker, thus strengthening their result for the viewing angle. Indeed the only constraint on the jet orientation comes from Pian et al. (1996), who deduce $\theta \simeq 30^{\circ} \pm$ $6^{\circ}$ from SSC models. We should also note that PKS 0521-36 seems to oscillate between a Seyfert-like and a BL Lac state (e.g. Ulrich 1981), making this source difficult to interpret within a purely non-thermal scenario, especially since we are confronted with non-simultaneous data.

BeppoSAX observed PKS 0521-36 on October 10, 1998 (Giommi et al. 2002) (black points in Fig. 8), and the Swift/XRT measurements (green points) taken on May 26, 2005 were obtained through the Online Analysis Tool ${ }^{5}$. The data points in gray are from the NED. We report in red the upper limit at $2 \sigma$ obtained in $89 \mathrm{~h}$ from observations by CANGAROO between 1993 and 1996 (Roberts et al. 1998). We used the EGRET data between $30 \mathrm{MeV}$ and $500 \mathrm{MeV}$ from Hartman et al. (1999) and taken between July 12, 1994 and August 01, 1994. The blazar PKS 0521-36 is associated with the source 2EG J0524-3630 in the Second EGRET Catalog (Thompson et al. 1995), but during cycle 4 this source was found to lie outside the $99 \%$ confidence contour of EGRET. However, like Foschini et al. (2006), we assume in this work the identification with PKS 0521-36 to be valid, which is also pointed out by Tornikoski et al. (2002).

Figure 8 presents the SED of PKS 0521-36 with the anticipated VHE emission (see Col. 8 in Table 1 for the corresponding parameters). It seems unlikely that the X-rays are due to inverse Compton radiation since the inverse Compton scattering would

\footnotetext{
5 See http://www.asdc.asi.it
}

be with photons from the radio/optical contribution, which is not very variable, coming from an extended part of the jet. Since the X-rays show a high degree of variability, they most certainly come from a compact region and have a synchrotron origin. If the X-ray emission is truly due to the synchrotron process, we predict that this BL Lac object should be marginally detectable by the present HESS telescope array, and easily detectable by HESS II and by the next generation of Čerenkov arrays, such as the CTA project, which will detect sources down to $\sim 0.1 \%$ of the Crab flux. Furthermore, if PKS 0521-36 remains undetected at VHE, a misidentification with the EGRET source should be considered.

\section{Discussion and implications on the AGN unification scheme}

When applied to AGNs belonging to very different classes, the multi-blob model deduces very similar properties for the size of the $\mathrm{TeV}$ emitting zones and the values of the magnetic field. Furthermore, the inferred bulk Lorentz factors $\Gamma_{b}$ can usually remain below a value of 10 , thus reconciling SSC models with (GR)MHD models, except for the interpretation of the $\gamma$-ray bump of Cen A in terms of synchrotron emission (see the green lines in Fig. 7). A rather unified picture therefore seems to come out from the analysis.

In our scenario, we locate the $\mathrm{X}$ - and $\gamma$-ray emitting region in the jet-formation zone, with a larger opening angle than in the global VLBI radio zone, which is more distant from the core and mainly located in a region where the jet is more collimated. This is somewhat reminiscent of a proposal made several years ago by Celotti et al. (1993) for unifying X-ray and radio-selected BL Lac objects. Celotti et al. (1993) showed that such a picture can be coherent with statistics of the BL Lac population.

Since our model accounts for the observation with rather low bulk Lorentz factors and with large effective opening angles, we have found a way out of the problem of statistics for the number of detected TeV sources invoked by Henri \& Saugé (2006), and we can reconcile beamed and unbeamed sources. One important consequence of our proposal is that bright radio BL Lacs should be TeV emitters. This can be tested by further observations.

Indeed, Padovani (2007) shows that the common historical unified LBL/HBL scheme (the so-called "blazar sequence") seems to be ruled out by the discovery of "outlier" low-power LBL and high-power HBL sources. Hence it does not seem impossible that objects that are very different at first sight, like radiogalaxies such as Cen $\mathrm{A}$, could be (faint) VHE emitters. Furthermore, that M87 has already been detected in the TeV range is encouraging for future detection of such radiogalaxies. In the case of 3C 273, this object would more likely be a misaligned LBL-like object. However, one should stress that the fact that mainly HBL have been detected up to now at VHE is certainly only a selection effect. Thus a TeV detection of 3C 273 would not be very surprising.

The scenario we propose here has an additional interest in that it allows solution of the long-standing paradox of the apparent absence of high superluminal motion at the base of radio jets of TeV BL Lacs. In our model, some X- and $\gamma$-ray emitting plasma blobs are moving close to the line of sight, thus allowing Cen A and PKS 0521-36, for instance, to be potentially seen at VHE, while standing in misaligned extended jets.

This statement agrees well with the most recent studies by Gopal-Krishna et al. (2006, 2007), who show that viewing angles, opening angles, and Lorentz factors of (sub-)parsec scale 
jets evaluated by radio observations are usually underestimated, thus reconciling absent superluminal motions inferred from radio observations with high Lorentz factors required by different families of $\mathrm{TeV}$ emission models. Wiita (2006) also underlines the possibility of reconciling various contradictory observations by considering jets with opening angles of a few degrees. Early studies by Dermer \& Gehrels (1995) of $\gamma$-ray observations of AGNs with EGRET also indicate that strong beaming is not required to account for $\mathrm{TeV}$ blazar observations.

Two different variability time scales appear within our mutliblob scenario. The short time scale is related to the characteristic size of individual blobs, as already discussed. A longer time scale occurs in the case of rotating jets with a helicoidal magnetic field. This induces a rotation of the cap, and the lag between "on-blob" and "inter-blob" emission corresponds to a long-term $\gamma$-ray variability. From MHD models, we infer a characteristic rotation time of the magnetic field on the order of one year in the observer frame for M 87. This modulation could explain the variation on the VHE emission observed between low and high states in 2004 and 2005. This would imply some periodicity in the $\mathrm{TeV}$ emission of AGNs, but on timescales hitherto not explored.

\section{Conclusion}

We have presented an SSC model to interpret VHE emission of M 87, as well as other misaligned sources with an extended optical/X-ray jet. This model accounts in a simple way for a differential Doppler-boosting effect by modeling the emission of several blobs of plasma located in the broadened formation zone of the jet close to the SMBH, just beyond the Alfvén surface predicted by MHD models.

Our scenario provides a reasonable interpretation of the HESS VHE observations of M 87 and provides the possibility of extending standard leptonic models of TeV blazars to other types of AGNs. However, we do not exclude other leptonic or hadronic models. For instance, Neronov \& Aharonian (2007) have recently interpreted the HESS observations from 2005 of M 87 by invoking acceleration and radiation of electrons in the black hole magnetosphere, which is another kind of leptonic model. Hadronic models also cannot be excluded, since efficient particle acceleration processes can occur in the close surroundings of the black hole.

More observations are needed to constrain the emission models and especially to distinguish between hadronic and leptonic scenarii. The upcoming GLAST mission and the HESS II project will certainly help in understanding the mechanisms at work in the AGNs by exploring spectral ranges below $\mathrm{TeV}$, which is decisive for constraining the shape of the inverse Compton bump. Moreover, the gap in genuine simultaneous multiwavelength campaigns on M 87 needs to be filled, especially since this source is known to be variable on small timescales in VHE.

Several types of active nuclei are potential emitters of VHE photons with predicted $\mathrm{TeV}$ fluxes detectable by present Čerenkov arrays like HESS and MAGIC, or by the next generation of instruments such as the CTA project. Such data will be crucial for testing AGN unifying schemes.

Acknowledgements. We are grateful to the anonymous referee for useful comments. J.-P. L. would like to thank Dr. A. Djannati-Ataï, Dr. S. Pita, and Dr. A. Zech for useful discussions.
This research made use of the NASA/IPAC Extragalactic Database (NED), which is operated by the Jet Propulsion Laboratory, California Institute of Technology, under contract with the National Aeronautics and Space Administration.

\section{References}

Aharonian, F., Akhperjanian, A., Beilicke, M., et al. (HEGRA Collab.) 2003, A\&A, 403, L1

Aharonian, F., Akhperjanian, A. G., Bazer-Bachi, A. R., et al. (HESS Collab.) 2005, A\&A, 441, 465

Aharonian, F., Akhperjanian, A. G., Bazer-Bachi, A. R., et al. (HESS Collab.) 2006, Science, 314, 1424

Antonucci, R. 1993, ARA\&A, 31, 473

Bai, J. M., \& Lee, M. G. 2001, ApJ, 549, L173

Beilicke, M., Benbow, W., Cornils, R., et al. 2005, [arXiv: astro-ph/0504395]

Beilicke, M., Götting, N., \& Tluczykont, M. 2004, New Astron. Rev., 48, 407 Berghöfer, T. W., Bowyer, S., \& Korpela, E. 2000, ApJ, 535, 615 Biretta, J. A., Stern, C. P., \& Harris, D. E. 1991, AJ, 101, 1632

Biretta, J. A., Sparks, W. B., \& Macchetto, F. 1999, ApJ, 520, 621

Biretta, J. A., Junor, W., \& Livio, M. 2002, New Astron. Rev., 46, 239

Bloom, S. D., \& Marscher, A. P. 1996, ApJ, 461, 657

Böhringer, H., Belsole, E., Kennea, J., et al. 2001, A\&A, 365, L181

Burns, J. O., Feigelson, E. D., \& Schreier, E. J. 1983, ApJ, 273, 128

Capetti, A., Trussoni, E., Celotti, A., Feretti, L., \& Chiaberge, M. 2000, MNRAS, 318,493

Celotti, A., Maraschi, L., Ghisellini, G., Caccianiga, A., \& Maccacaro, T. 1993, ApJ, 416, 118

Chiaberge, M., \& Ghisellini, G. 1999, MNRAS, 306, 551

Chiaberge, M., Capetti, A., \& Celotti, A. 2001, MNRAS, 324, L33

Collmar, W., Reimer, O., Bennett, K., et al. 2000, A\&A, 354, 513

Dermer, C. D., \& Gehrels, N. 1995, ApJ, 447, 103

Evans, D. A., Kraft, R. P., Worrall, D. M., et al. 2004, ApJ, 612, 786

Fioc, M., \& Rocca-Volmerange, B. 1997, A\&A, 326, 950

Foschini, L., Ghisellini, G., Raiteri, C. M., et al. 2006, A\&A, 453, 829

Fossati, G., Maraschi, L., Celotti, A., Comastri, A., \& Ghisellini, G. 1998, MNRAS, 299, 433

Georganopoulos, M., Perlman, E. S., \& Kazanas, D. 2005, ApJ, 634, L33

Georganopoulos, M., Perlman, E. S., Kazanas, D., \& McEnery, J. 2006, ApJ, 653, L5

Ghisellini, G., Celotti, A., Fossati, G., Maraschi, L., \& Comastri, A. 1998, MNRAS, 301, 451

Giommi, P., Capalbi, M., Fiocchi, M., et al. 2002, in Blazar Astrophysics with BeppoSAX and Other Observatories, ed. P. Giommi, E. Massaro, \& G. Palumbo, 63

Gopal-Krishna, Dhurde, S., Sircar, P., \& Wiita, P. J. 2007, MNRAS, 377, 446

Gopal-Krishna, Wiita, P. J., \& Dhurde, S. 2006, MNRAS, 369, 1287

Gould, R. J. 1979, A\&A, 76, 306

Graham, J. A. 1978, PASP, 90, 237

Grandi, P., \& Palumbo, G. G. C. 2007, ApJ, 659, 235

Grandi, P., Malaguti, G., \& Fiocchi, M. 2006, ApJ, 642, 113

Hardcastle, M. J., Worrall, D. M., Kraft, R. P., et al. 2003, ApJ, 593, 169

Häring-Neumayer, N., Cappellari, M., Rix, H.-W., et al. 2006, ApJ, 643, 226

Hartman, R. C., Bertsch, D. L., Bloom, S. D., et al. 1999, ApJS, 123, 79

Hawarden, T. G., Sandell, G., Matthews, H. E., et al. 1993, MNRAS, 260, 844

Henri, G., \& Saugé, L. 2006, ApJ, 640, 185

Horiuchi, S., Meier, D. L., Preston, R. A., \& Tingay, S. J. 2006, PASJ, 58, 211

Inoue, S., \& Takahara, F. 1996, ApJ, 463, 555

Katarzyński, K., Sol, H., \& Kus, A. 2001, A\&A, 367, 809

Katarzyński, K., Sol, H., \& Kus, A. 2003, A\&A, 410, 101

Keel, W. C. 1985, AJ, 90, 2207

Kinzer, R. L., Johnson, W. N., Dermer, C. D., et al. 1995, ApJ, 449, 105

Le Bohec, S., Badran, H. M., Bond, I. H., et al. 2004, ApJ, 610, 156

Macchetto, F., Marconi, A., Axon, D. J., et al. 1997, ApJ, 489, 579

Marconi, A., Schreier, E. J., Koekemoer, A., et al. 2000, ApJ, 528, 276

Marconi, A., Pastorini, G., Pacini, F., et al. 2006, A\&A, 448, 921

McKinney, J. C. 2006, MNRAS, 368, 1561

Melia, F., Liu, S., \& Fatuzzo, M. 2002, ApJ, 567, 811

Mirabel, I. F., Laurent, O., Sanders, D. B., et al. 1999, A\&A, 341, 667

Neronov, A., \& Aharonian, F. 2007, [arXiv: 0704.3282]

Padovani, P. 2007, Ap\&SS, 309, 63

Paltani, S., \& Türler, M. 2005, A\&A, 435, 811

Perlman, E. S., \& Wilson, A. S. 2005, ApJ, 627, 140 
Perlman, E. S., Sparks, W. B., Radomski, J., et al. 2001, ApJ, 561, L51

Perlman, E. S., Harris, D. E., Biretta, J. A., Sparks, W. B., \& Macchetto, F. D 2003, ApJ, 599, L65

Pian, E., Falomo, R., Ghisellini, G., et al. 1996, ApJ, 459, 169

Reynolds, C. S., Fabian, A. C., Celotti, A., \& Rees, M. J. 1996, MNRAS, 283, 873

Roberts, M. D., Dazeley, S. A., Edwards, P. G., et al. (CANGAROO Collab.) 1998, A\&A, 337, 25

Rothschild, R. E., Wilms, J., Tomsick, J., et al. 2006, ApJ, 641, 801

Schmidt, M. 1963, Nature, 197, 1040

Smith, R. J., Lucey, J. R., Hudson, M. J., Schlegel, D. J., \& Davies, R. L. 2000, MNRAS, 313, 469

Sparks, W. B., Biretta, J. A., \& Macchetto, F. 1996, ApJ, 473, 254

Sreekumar, P., Bertsch, D. L., Dingus, B. L., et al. 1994, ApJ, 426, 105

Stawarz, Ł., Sikora, M., \& Ostrowski, M. 2003, ApJ, 597, 186

Stawarz, Ł., Aharonian, F., Wagner, S., \& Ostrowski, M. 2006, MNRAS, 371, 1705

Stecker, F. W., Malkan, M. A., \& Scully, S. T. 2006, ApJ, 648, 774
Steinle, H., Bennett, K., Bloemen, H., et al. 1998, A\&A, 330, 97

Strauss, M. A., Huchra, J. P., Davis, M., et al. 1992, ApJS, 83, 29

Thompson, D. J., Bertsch, D. L., Dingus, B. L., et al. 1995, ApJS, 101, 259

Tingay, S. J., \& Edwards, P. G. 2002, AJ, 124, 652

Tingay, S. J., Jauncey, D. L., Reynolds, J. E., et al. 1998, AJ, 115, 960

Tornikoski, M., Lähteenmäki, A., Lainela, M., \& Valtaoja, E. 2002, ApJ, 579, 136

Türler, M., Paltani, S., Courvoisier, T. J.-L., et al. 1999, A\&AS, 134, 89

Türler, M., Chernyakova, M., Courvoisier, T. J.-L., et al. 2006, A\&A, 451, L1

Ulrich, M. H. 1981, A\&A, 103, L1

Unwin, S. C., Cohen, M. H., Biretta, J. A., et al. 1985, ApJ, 289, 109

Urry, C. M., \& Padovani, P. 1995, PASP, 107, 803

Vlahakis, N., \& Königl, A. 2004, ApJ, 605, 656

Wiita, P. J. 2006, [arXiv: astro-ph/0603728]

Wilson, A. S., \& Yang, Y. 2002, ApJ, 568, 133

Woo, J.-H., Urry, C. M., van der Marel, R. P., Lira, P., \& Maza, J. 2005, ApJ, 631,762 